\title{
Wavelength-stabilized tapered laser diodes in an external Talbot cavity: simulations and experiments
}

\author{
David Paboeuf*a, Gaëlle Lucas-Leclin ${ }^{\mathrm{a}}$, Patrick Georges ${ }^{\mathrm{a}}$, Nicolas Michel ${ }^{\mathrm{b}}$, Michel Krakowski ${ }^{\mathrm{b}}$, \\ Jun Jun Lim ${ }^{c}$, Slawomir Sujecki ${ }^{c}$, Eric Larkins ${ }^{c}$ \\ a'Laboratoire Charles Fabry de L'Institut d'Optique, CNRS, Univ Paris-Sud, Campus Polytechnique, \\ RD128, 91127 Palaiseau Cedex, France \\ ${ }^{\mathrm{b}}$ Alcatel-Thales III-V Lab, RD128, 91767 Palaiseau - France \\ ${ }^{c}$ The School of Electronic and Electrical Engineering, University of Nottingham, University Park, \\ Nottingham NG7 2RD - UK
}

\begin{abstract}
We present the experimental and theoretical evaluation of the coherent combining of an array of index-guided tapered laser diodes in an external Talbot cavity. A theoretical model taking into account the propagation inside the semiconductor device has been developed to determine the cavity spatial modes. In parallel, experiments have been realized with 10 emitters in a compact setup and a volume Bragg grating as the external mirror. $1.7 \mathrm{~W}$ have been obtained at $976 \mathrm{~nm}$ for the in-phase mode in a narrow laser line $(<0.1 \mathrm{~nm})$.

Keywords: Bragg gratings, semiconductor laser array, Talbot effect, laser modeling
\end{abstract}

\section{INTRODUCTION}

High power laser diode arrays are very attractive for applications such as solid-state laser pumping or laser material processing due to a very good overall efficiency and compactness. Inconveniently, the beam quality and the spectrum bandwidth of these devices are often too far from the requirements for demanding applications. A well-known way to enhance the beam quality of a group of many lasers is to induce a phase locking between them. Numerous techniques based on external cavities have been developed in the past with fiber lasers [1], $\mathrm{CO}_{2}$ lasers [2] and laser diodes [3]. Among all these techniques, the use of the self-imaging Talbot effect has been established as a very efficient way to produce a highly coherent emission of an array of laser diodes, demonstrating output powers in the Watt range in the inphase mode [4-6]. However the brightness performance of these lasers is limited by several factors: the free-running operation of the emitters which limits the operating current range for efficient phase locking [7]; the use of intracavity filters and magnification optics which induces extra losses [4]; and the beam quality of the emitters by themselves. Moreover, the spectrum is often very unstable [6] and may contribute to the coherency degradation observed at high current. The wavelength stabilization of the laser array can be obtained with a standard diffraction grating [8] but with the drawback of complexity. On the contrary in this work we propose to use a volume Bragg grating $[9,10]$ as the output coupler of an external cavity based on the Talbot self-imaging effect, in order to phase-lock an array of 10 tapered laser diodes and concurrently stabilize the laser spectrum. Moreover, we describe numerical simulations of the propagation of the optical beam inside the external-cavity diode laser array; these simulations take into account both the propagation into the semiconductor gain medium and in the external cavity. The results confirmed the strong discrimination between the modes of our laser cavity and allowed the identification of some effects that may limit the performance.

\footnotetext{
*david.paboeuf@institutoptique.fr
} 


\section{NUMERICAL MODELING OF THE EXTERNAL CAVITY OPERATION}

\subsection{Laser diode array design}

The laser source is an array of 10 index-guided tapered laser diodes with a pitch $\mathrm{p}=100 \mu \mathrm{m}$, which results in a Talbot distance of $20.4 \mathrm{~mm}$. The active layer consists of a strained GaInAs quantum well embedded in a large optical cavity. The lateral structure of the emitters is a tapered ridge with a narrow angle $\left(<1^{\circ}\right)$ and an overall cavity length of $2.5 \mathrm{~mm}$. The tapered design allows high power emission around $975 \mathrm{~nm}$ together with single transverse mode operation thanks to the widened active section. The large pitch between the emitters forbid any evanescent coupling between adjacent emitters, thus the beam from the whole array is incoherent with a divergence far from the diffraction limit. Such an array has already demonstrated $4 \mathrm{~W}$ at $6 \mathrm{~A}$ [9] with an incoherent far field full-width at $1 / \mathrm{e}^{2}$ in the slow axis of $2.7^{\circ}$ and a $\mathrm{M}^{2}$ parameter $<2$. The rear facet is high-reflection coated $(\mathrm{R}>90 \%)$. Thanks to the antireflection coating $\left(\mathrm{R}<10^{-3}\right)$ on the front facet of the laser array, no laser emission but only amplified spontaneous emission (ASE) is observed without external cavity. This feature is very important to avoid competition between the free-running operation of the emitters and the coherent emission induced by the external cavity.

\subsection{Talbot cavity}

Optical coupling between $\mathrm{N}$ adjacent lasers leads to $\mathrm{N}$ array supermodes corresponding to the $\mathrm{N}$ different coherent optical field repartitions along the bar. These modes are the eigenmodes of the laser cavity. Following Butler et al. [10], these profiles can be expressed as :

$$
E_{n}(x)=\sum_{m=1}^{N} \sin \left(m \frac{n \pi}{N+1}\right) u_{m}(x),
$$

where $\mathrm{n}$ is the mode number and $\mathrm{u}_{\mathrm{m}}$ stands for the profile of the $\mathrm{m}^{\text {th }}$ laser. Among these modes, the preferred one is the first order mode also called in-phase mode, where each laser has the same phase, since its far-field profile exhibits a central high intensity main lobe (see Fig. 4).

The propagation of an infinite coherent periodic field results in the apparition of self-images (in amplitude and phase) of the initial field every integer multiple of the distance $Z_{T}=2 p^{2} / \lambda$. This pure diffractive phenomenon is called Talbot effect [11] and $Z_{T}$ is the so called Talbot distance. Image-like patterns also appear at fractional Talbot distances $Z_{T} / n$. In particular at $\mathrm{z}=\mathrm{Z}_{\mathrm{T}} / 2$, the out-of-phase $(\mathrm{n}=\mathrm{N})$ mode is exactly self-imaged; at that distance the in-phase mode (n=1) is also self-imaged but with a lateral shift of $\mathrm{p} / 2$. This results in a maximal discrimination of these two very modes. For arrays with finite dimensions, the Talbot effect can also be observed, but edge losses appear that slightly degrade the imaging quality. This is obvious on Fig. 1 where the propagation of 10 coherent Gaussian-shaped emitters has been computed using Fresnel integral [12]. At $\mathrm{z}=2 \mathrm{Z}_{\mathrm{T}}$, the edges losses are so high that self-images are barely observable.
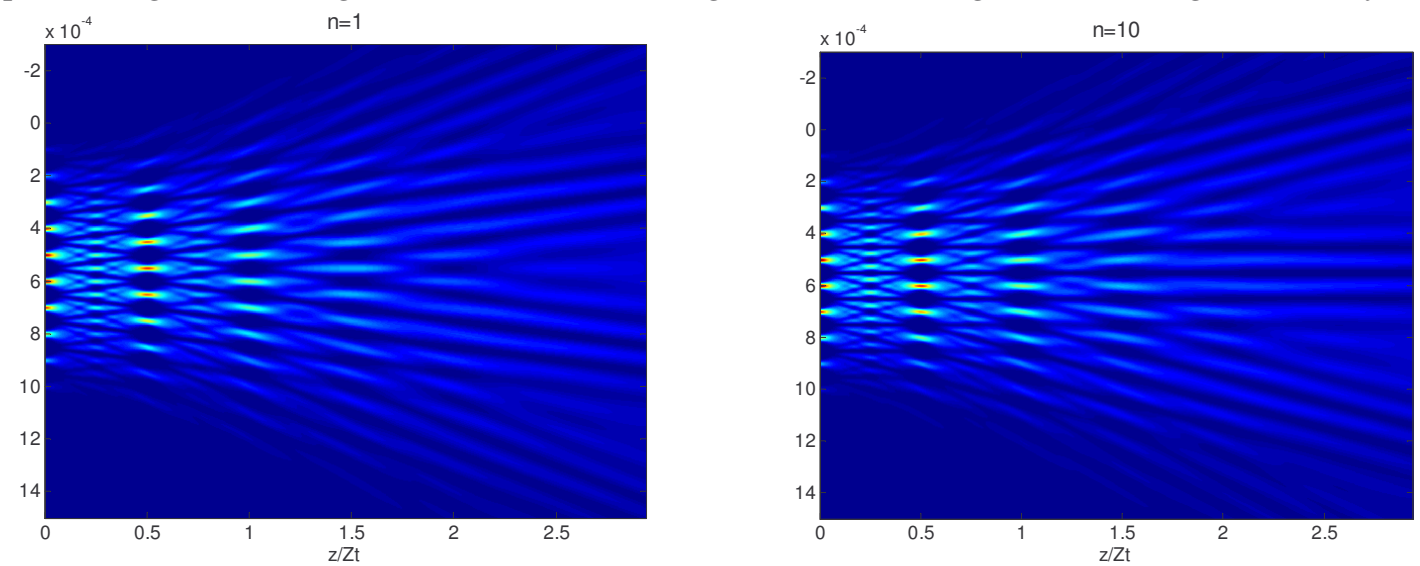

Fig. 1: Propagation of the in-phase (left) and out-of-phase (right) modes for 10 Gaussian emitters $(\mathrm{p}=100 \mu \mathrm{m}$, width = $10 \mu \mathrm{m})$

Talbot laser cavities utilize the Talbot effect to force the laser array into a coherent emission. Indeed, the reflection into the emitters of the optical field $\mathrm{E}\left(\mathrm{L}_{\mathrm{ext}}\right)$, which has been propagated back and forth on a distance $\mathrm{Z}_{\mathrm{T}}$ in the external cavity of length $\mathrm{L}_{\mathrm{ext}}=\mathrm{Z}_{\mathrm{T}} / 2$, would induce a coupling between adjacent emitters and would favor a coherent emission of the 
whole array. However as the in-phase and out-of-phase modes would both be self-imaged, no discrimination would be possible between these supermodes, and the laser emission would thus be spatially multimode. On the contrary a cavity length $\mathrm{L}_{\mathrm{ext}}=\mathrm{Z}_{\mathrm{T}} / 4$ would allow to strongly discriminate the in-phase and out-of-phase modes of the array thanks to the $\mathrm{p} / 2$ lateral shift of the Talbot image at $\mathrm{z}=\mathrm{Z}_{\mathrm{T}} / 2$. The preferred in-phase mode would be simply selected by a tilted mirror compensating the lateral shift on roundtrip propagation.

\subsection{Model description}

Simulations of the behavior of the cavity have been carried out with an iterative approach based on the Fox-Li method. The optical beam is propagated back and forth inside the cavity until a steady state power and amplitude profile are reached. The model of the tapered laser bar combines the electrical and optical models in the same way as described by Lim et al. [13]. The isothermal electrical model consists of the 1D unipolar carrier-diffusion equation. A constant injection current density $\mathrm{J}(\mathrm{x})$ is assumed inside the stripe. The carrier density distribution is solved so that spatial hole burning and carrier lensing effects are included. The optical fields in the tapered laser are modeled with the 2D wideangle finite difference beam propagation method (WA-FDBPM) using the effective index approximation. The free-space propagation is modeled using the Fresnel diffraction equations [12] and includes reflection from a tilted plane output mirror, in the external cavity. We assumed a TE polarized and monochromatic optical beam.

An intensity map of the in-phase mode inside the tapered semiconductor laser array is presented in Fig. 2. The beam propagation inside the emitters is not straight and exhibits some reflections on the side of the waveguides. These reflections may induce some extra-losses inside the laser cavity. This effect is stronger for the bottom emitters and is related to the tilt angle of the external mirror.

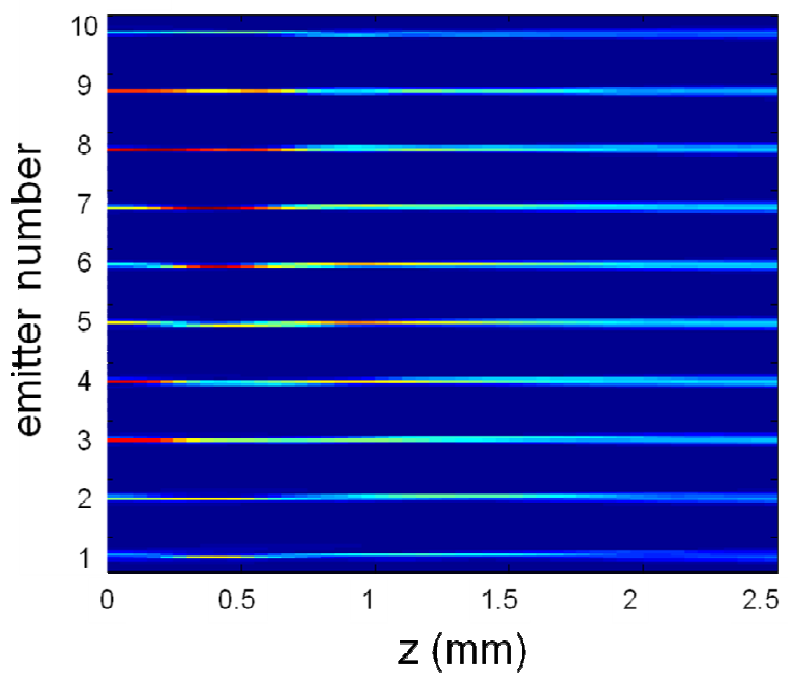

Fig. 2: Intensity map of the in-phase mode inside the semiconductor gain medium (I=3.88A)

\subsection{Gain-Loss characterization}

In order to evaluate the influence of the slight degradation of the Talbot self-imaging on the propagation of the beams inside the emitters under external cavity operation, we have calculated the optical gain inside each laser. The gain inside each emitter was evaluated as the ratio between the power of the incoming beam into the laser on the tapered side and the power of the output beam after round-trip propagation inside the gain medium:

$$
G_{k}=\frac{\int_{\text {emitter } k} I_{\text {out }}(x) d x}{\int_{\text {emitter } k} I_{\text {in }}(x) d x},
$$

where $\mathrm{k}$ stands for the emitter number. As $G_{k}$ is computed above threshold, its value is directly proportional to the coupling losses induced by the external cavity. The results for both in-phase and out-of-phase modes are shown in Fig. 3 for an operating current of $3.88 \mathrm{~A}$. As it should be expected with a symmetrical cavity, the gain repartition is symmetric for the out-of-phase mode with a maximum at both edges which is correlated to the higher propagation losses for 
marginal emitters that have been evidenced in Fig. 2. These losses have been estimated to be $40 \%$ higher than at the center. For the in-phase mode, the gain repartition is asymmetric with maximum losses at the left border of the array, corresponding to the tilt direction of the mirror. The maximal losses are $90 \%$ higher than for the emitter at the middle of the array. These extra-losses result in a higher threshold and a slightly lower output power for the in-phase mode as compared to the out-of-phase mode.
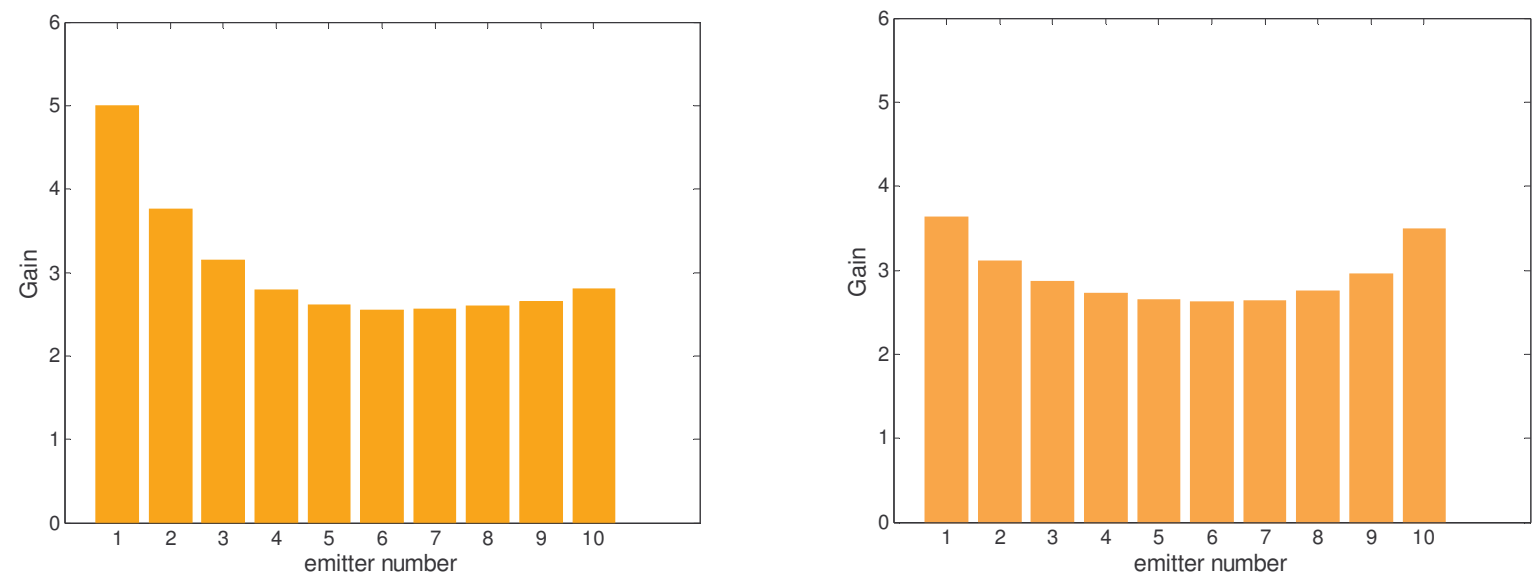

Fig. 3: Gain distribution of each emitter (left - in-phase mode, right - out-of-phase mode)

\subsection{Modal discrimination of the cavity}

We have first checked the behavior of the cavity and the modal selection evolution with the tilt of the mirror. The reflectivity of the mirror was set to $40 \%$ which corresponds to the effective reflectivity of the VBG used in the experiments. With a normal incidence mirror, the out-of phase mode oscillates inside the cavity whereas with a tilt angle of $\lambda / 2 p$, the in-phase mode is selected. The strong modal selectivity of the Talbot cavity is then confirmed.

The near field intensity profile of the in-phase mode (Fig. 4.) presents a slight asymmetry attributed to the tilt of the external mirror. On the other hand, the out-of-phase mode is very symmetric and remains close to the theoretical profile proposed by Butler et al [10]. The phase profiles exhibit some differences from one emitter to the other showing some phase mismatching. This distortion seems to be stronger at the array edges but remains included in a 0.5 rad range. It may be a consequence of the degradation of the self-imaged fields due to the finite size of the laser array. 
In-phase mode

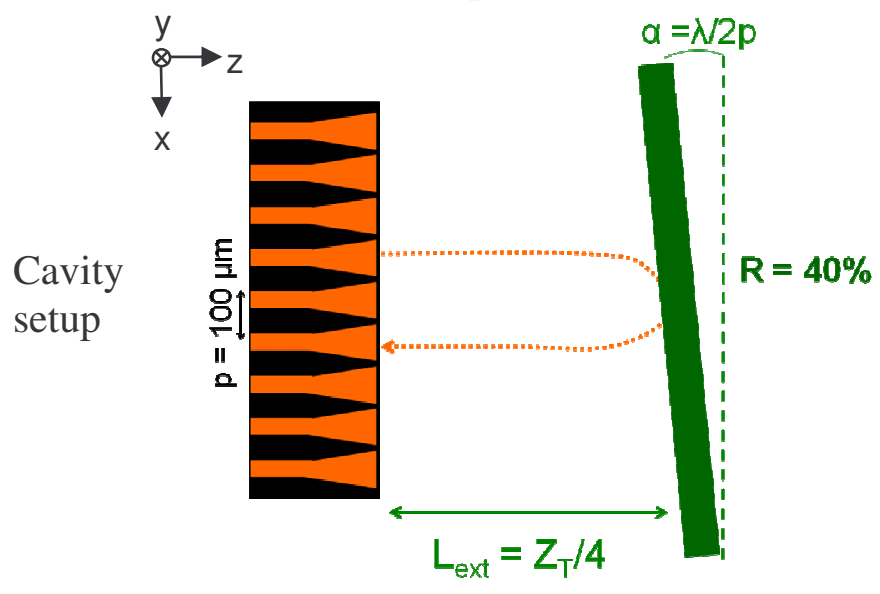

Near-

field

profile

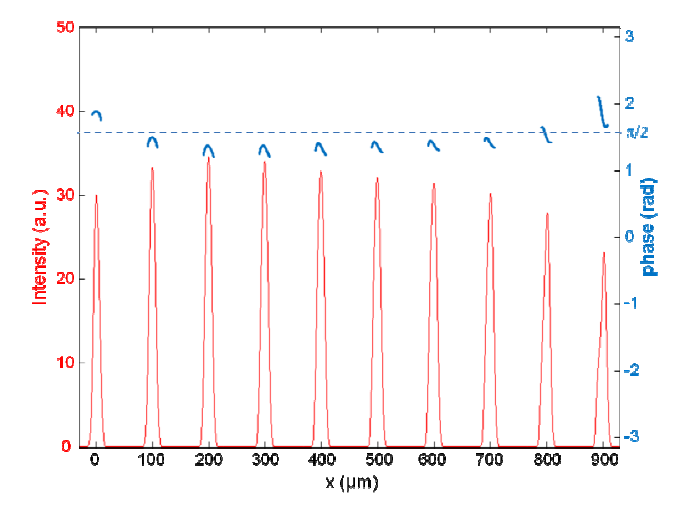

Far-

field

profile

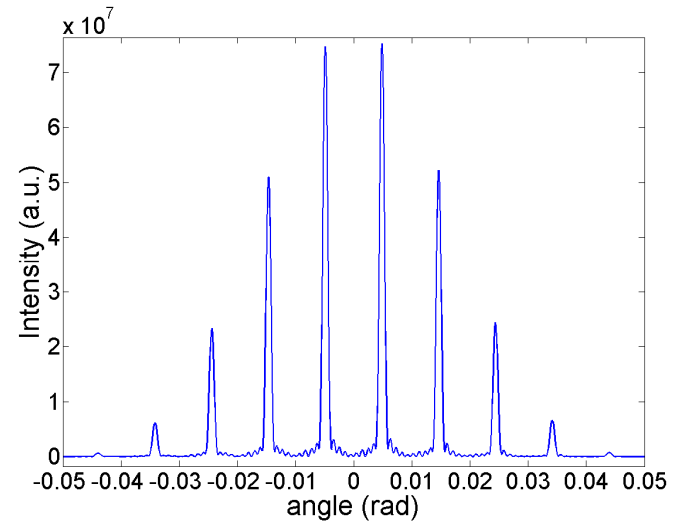

Out-of-phase mode
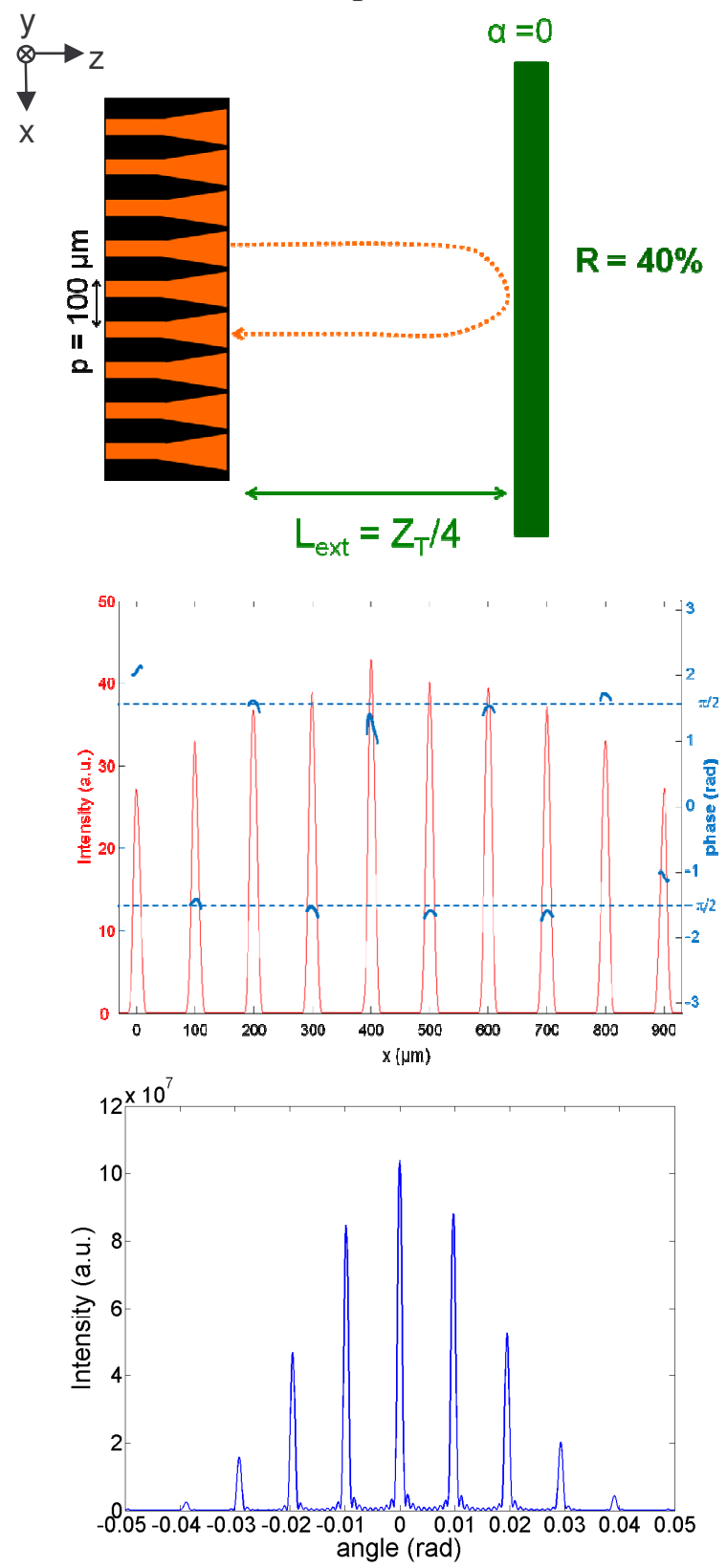

Fig. 4: Numerical near-field and far-field profiles of the modes of the external Talbot cavity at I $=3.88 \mathrm{~A}$ (left - in phase mode, right - out-of-phase mode)

\section{EXPERIMENTAL RESULTS}

\subsection{Experimental setup}

Our experimental setup is shown in Fig. 5. The beam from the laser array is collimated in the fast axis direction with a high numerical aperture acylindric lens (Doric Lenses - D142-375). The remaining divergence is lower than $0.17^{\circ}$ $(3 \mathrm{mrad})$. During the alignment, the lens is fixed to a three-axis $(X Y Z)$ translation stage; then it is glued to its optimal position with an epoxy adhesive (Araldite) and the translation stage is removed. A volume Bragg grating from ONDAX, 
Inc is used as the external mirror. It is designed to reflect $40 \%$ at $976 \mathrm{~nm}$ with a spectral bandwidth $\Delta \lambda=0.3 \mathrm{~nm}$. The angular acceptance of the volume Bragg grating (VBG) is $\Delta \theta=3^{\circ}$ (full width at $1 / \mathrm{e}^{2}$ ), which is larger than the divergence of the laser array in both directions after fast axis collimation. The diffraction efficiency of the VBG will then remain close to maximum [14]. The VBG is located at a quarter of the Talbot distance from the front facet for maximum discrimination between the in-phase and the out-of-phase modes. This setup results in a very compact (5 mm-long) and simple cavity (see Fig. 6), due to the choice of the array pitch, with only one intracavity element for limited losses. The whole setup is mounted on a copper block and actively thermally regulated.

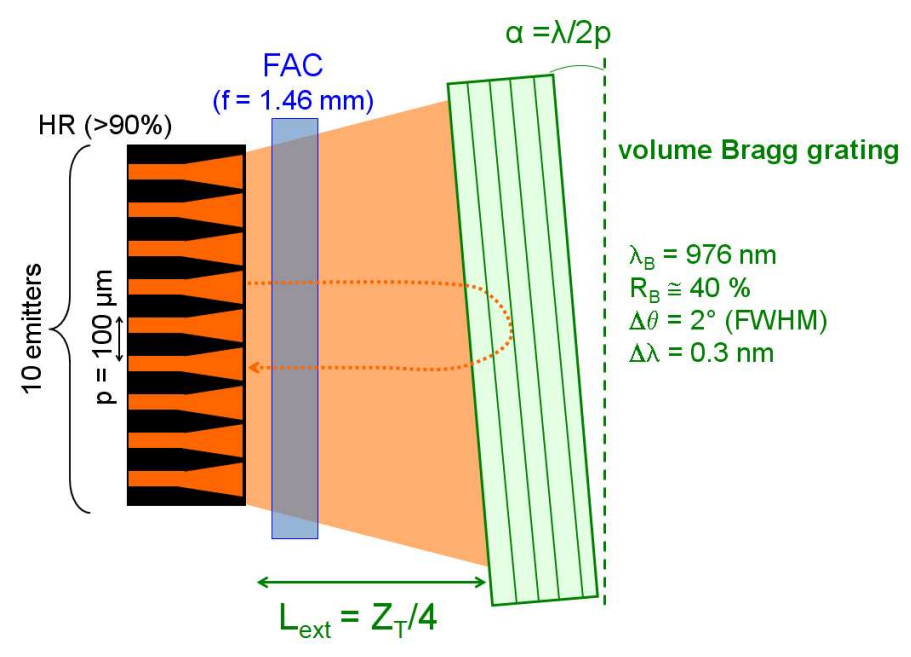

Fig. 5: Experimental setup of the external Talbot cavity

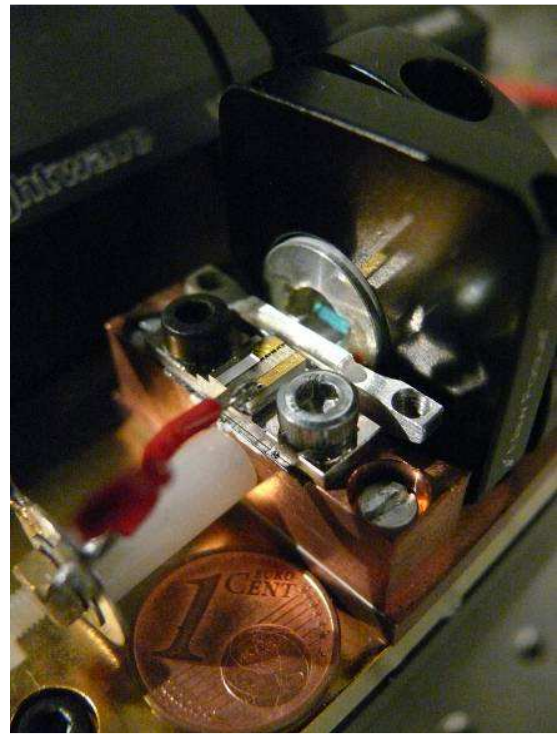

Fig. 6: Photograph of the laser cavity

\subsection{Power characterization}

With the VBG as the output coupler of the cavity, a threshold of $0.9 \mathrm{~A}$ and a power of $1.7 \mathrm{~W}$ at an operating current of 3.9 A have been obtained. The slope efficiency is $0.6 \mathrm{~W} \cdot \mathrm{A}^{-1}$ (see Fig. 7). With untilted VBG, the cavity operates in the out-of-phase mode. By tilting the VBG by an angle $\alpha=\lambda / 2 p$, the in-phase mode is selected. The maximum output power is only limited by the thermal roll-over of the array, which appears at $4 \mathrm{~A}$ under our experimental conditions. This maximum output power value roughly corresponds to $60 \%$ of the power obtained in free-running operation $(2.8 \mathrm{~W})$ at the same current with a similar chip [9], which means that the external cavity configuration extracts the maximum available power from the laser bar.

In Fig. 7, we compare the experimental L-I characteristic to the simulated one. For each current value we checked that the program converges to the in-phase supermode. Although the simulation results exhibit both higher threshold and slope efficiency, the two characteristics are in good agreement. The differences are attributed to disparities between the theoretical laser parameters used in the model and their effective values in the experiments. In addition, the use of a unipolar, isothermal model, which does not properly account for current spreading and self-heating effects, could also contribute to the discrepancy. 


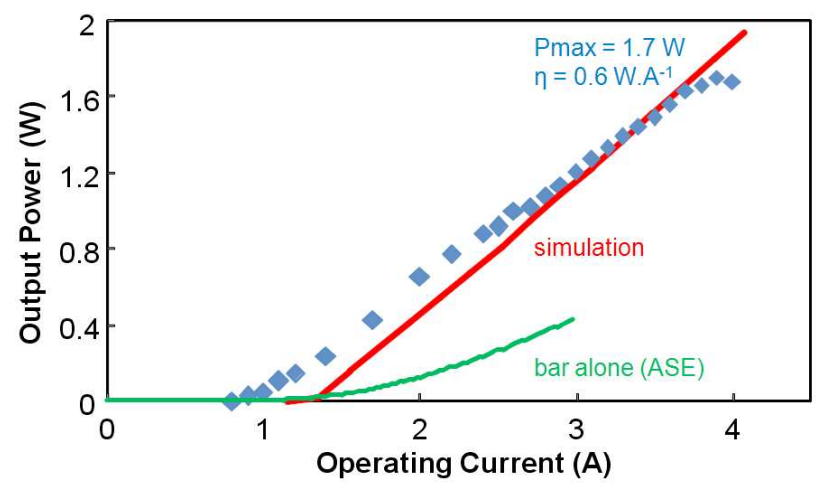

Fig. 7: L-I Characteristics of the external Talbot cavity (blue - experimental data, red - simulation data, green - laser bar alone (ASE))

\subsection{Far-field characterization}

The slow-axis angular distribution of the laser was measured with a CCD camera in the Fourier plane of a cylindrical collimating lens. The far-field profile exhibits interference peaks with a maximum at $\theta=0^{\circ}$, which is a clear evidence of the phase locking in the in-phase mode (see Fig. 8). The full width at half maximum of each peak is $1.2 \mathrm{mrad}$, which is very close to the diffraction angular limit of the array $(1 \mathrm{mrad})$. At high output powers $(\mathrm{P}>1.2 \mathrm{~W})$, the incoherent contribution of the free-running array operation induces a parasitic pedestal in the far-field distribution; moreover the interference peaks are slightly broadened to $2.5 \mathrm{mrad}$. In order to characterize the coherence quality of our cavity, we have calculated the visibility defined as follows:

$$
V=\frac{I_{\max }-I_{\min }}{I_{\max }+I_{\min }}
$$

A visibility as high as $80 \%$ has been measured at low power $(800 \mathrm{~mW}, \mathrm{I}=2.5 \mathrm{~A})$, which demonstrates the high degree of coherence induced by the Talbot external cavity. At higher currents, the visibility decreases to $70 \%$ due to increased incoherent free-running contribution.

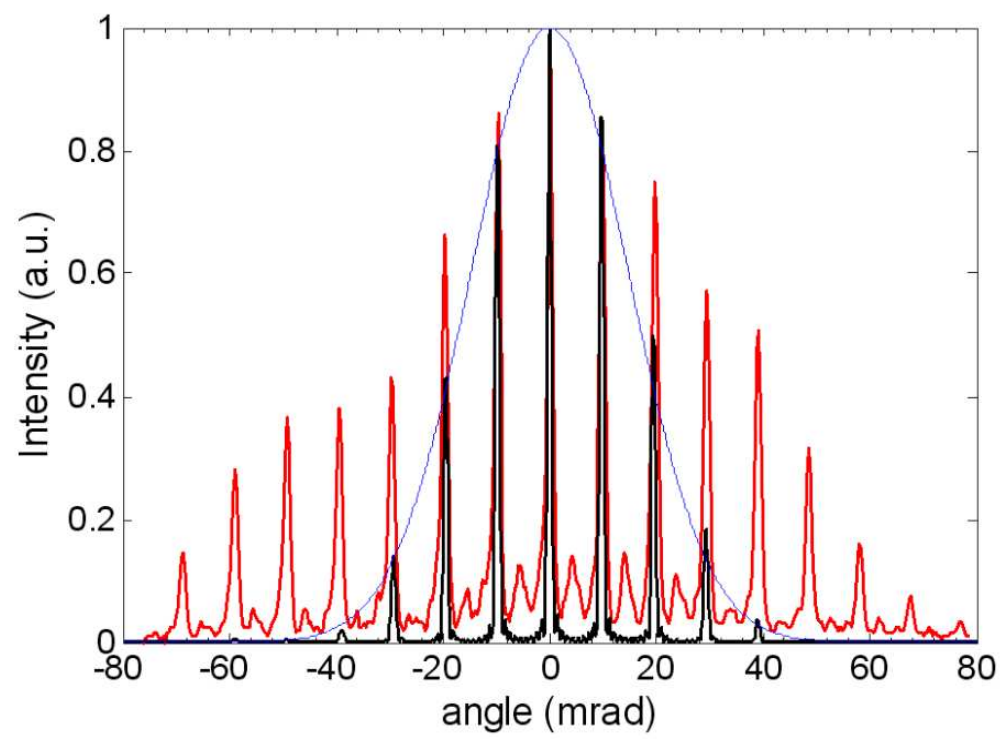

Fig. 8: Far field profile at I= 3 A (red - experimental measure, black - simulation, blue - Gaussian shape envelope) 
The envelope of the far field presents some distortion at high power, which is attributed to a degradation of the beam quality of the emitters. The far-field distribution obtained at $3 \mathrm{~A}$ is compared to the experimental one in Fig. 8. These results show that the interference peaks have the same width of $1.2 \mathrm{mrad}$ in both cases. The theoretical envelope of the far-field is close to a Gaussian profile with a full width at $1 / \mathrm{e}^{2}$ of $40 \mathrm{mrad}\left(2.3^{\circ}\right)$ related to the dimension of the emitters. It differs from the experimental one, which is larger, because of the presence of a multimode emission from the tapered diodes.

We have checked that similar performance is obtained with a plane mirror $(\mathrm{R}=40 \%)$ as the output coupler instead of the VBG, which shows the efficiency of the VBG to provide a coupling between the emitters together with a strong spectral control

\subsection{Wavelength Stabilization}

A spectrally resolved near field ( $\mathrm{SNeaF}$ ) has been measured by imaging the laser array near field at the entrance slit of a monochromator and a CCD camera at the output of the monochromator. The resulting spectra are presented in Fig. 9. It is clear on this figure that every emitter is locked to the Bragg wavelength. In fact, thanks to the VBG, the laser emission is only possible at the Bragg wavelength. The spectrum linewidth of the laser array has been further characterized with a high-resolution $(10 \mathrm{pm})$ optical spectrum analyzer; we evaluate the spectral linewidth to be below $0.1 \mathrm{~nm}$ whatever the operating current $(\mathrm{I}<4 \mathrm{~A})$ [15]. On the contrary with a mirror as the output coupler, the spectrum is very unstable and exhibits several lines for each emitter (see Fig. 10).

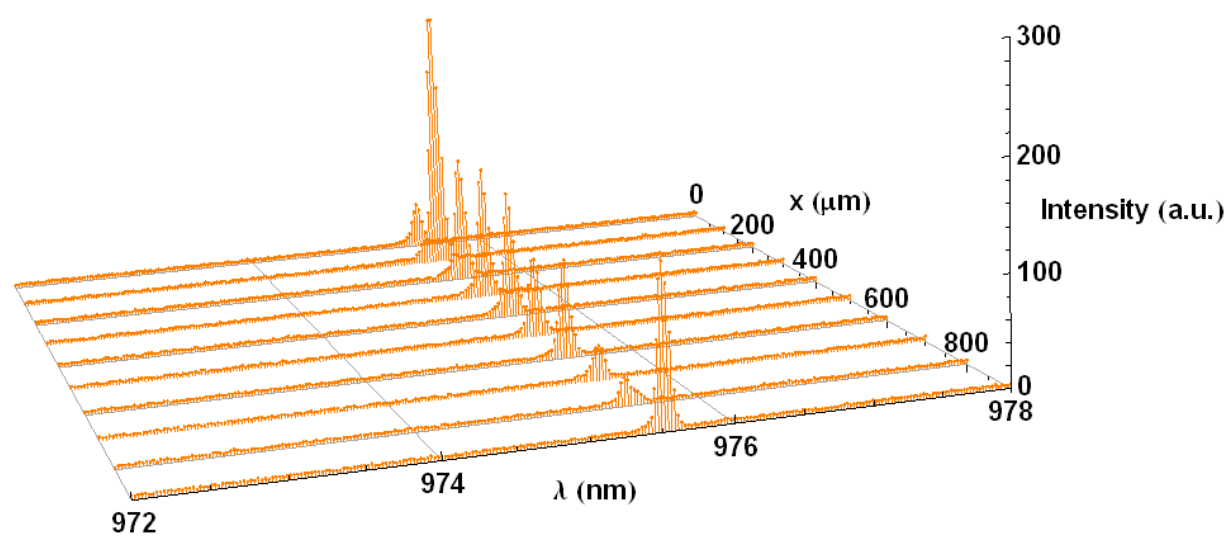

Fig. 9: SNeaF of the laser array in external cavity with a VBG at $\mathrm{I}=4 \mathrm{~A}, \mathrm{P}=1.7 \mathrm{~W}$

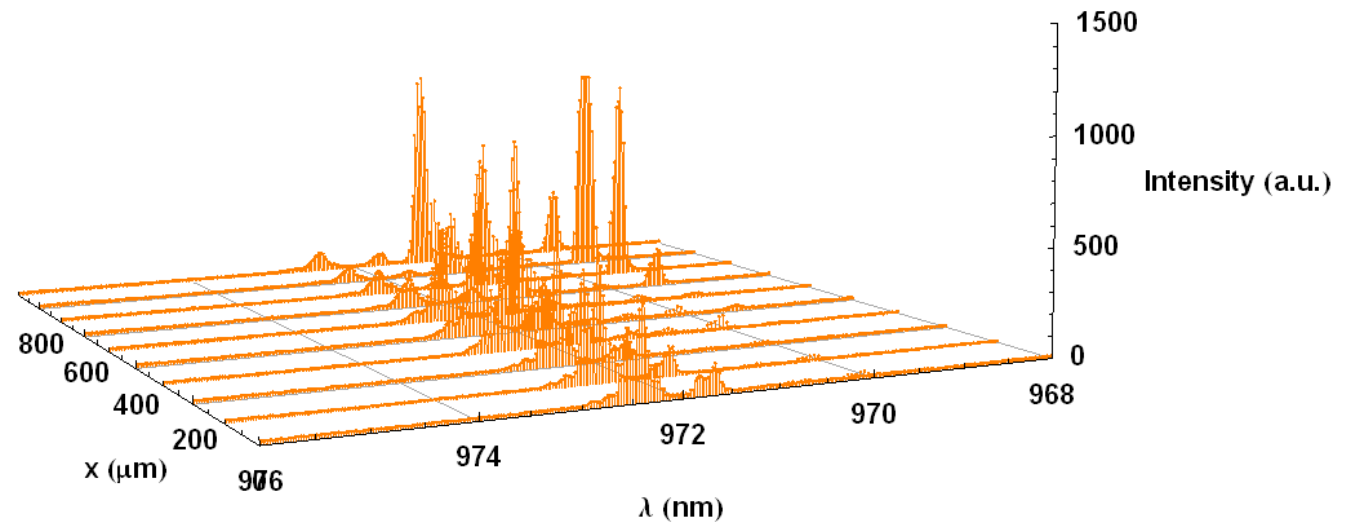

Fig. 10: Spectrally resolved near field $(\mathrm{SNeaF})$ of the laser array in external cavity with a plane mirror at $\mathrm{I}=2.1 \mathrm{~A}, \mathrm{P}=$ $200 \mathrm{~mW}$ 


\section{CONCLUSION}

In summary, we have demonstrated an original concept of external cavity exploiting the properties of volume Bragg gratings. A very simple and compact external cavity based on the Talbot self-imaging effect has been developed to perform the phase-locking of an array of tapered laser diodes. The careful design of the cavity parameters allows a highly coherent operation on a large power range. A maximum output power of $1.7 \mathrm{~W}$ is obtained in the in-phase array supermode. The wavelength of every emitter in the array is locked to the Bragg wavelength regardless of the operating conditions. A whole spectral bandwidth of $0.1 \mathrm{~nm}$ has been measured. In parallel, a numerical study taking into account the specific propagation inside the semiconductor medium has been carried out. It evidenced the strong modal discrimination of our cavity and gave access to the losses induced by the cavity which is a key point for an efficient laser operation. Increase of the power should be possible with improved laser diodes and a lower reflectivity grating. This concept could be easily adapted to other kinds of semiconductor laser arrays, such as gain-guided tapered laser diodes or Slab Coupled Optical Waveguide Lasers (SCOWLs) [6].

\section{ACKNOWLEDMENTS}

This work was supported within the European project BRIGHTER.EU under grant referenced FP6-IST-035266. D. Paboeuf thanks the French Ministry of Defense (Délégation Générale de l'Armement) for funding his PhD. N. Michel and M. Krakowski thank M. Calligaro, M. Lecomte, O. Parillaud, Y. Robert, M. Carbonnelle and C. Dernazaretian for the realization of tapered lasers.

\section{REFERENCES}

[1] C. J. Corcoran, and F. Durville, "Experimental demonstration of a phase-locked laser array using a self-Fourier cavity," Applied Physics Letters, 86(20), 201118 (2005).

[2] A. F. Glova, "Phase locking of optically coupled lasers," Quantum Electronics, 33(4), 283-306 (2003).

[3] C. J. Chang Hasnain, J. Berger, D. R. Scifres, W. Streifer, J. R. Whinnery, and A. Dienes, "High-Power with High-Efficiency in a Narrow Single-Lobed Beam from a Diode-Laser Array in an External Cavity," Applied Physics Letters, 50(21), 1465-1467 (1987).

[4] R. Waarts, D. Mehuys, D. Nam, D. Welch, W. Streifer, and D. Scifres, "High-Power, CW, Diffraction-Limited, GaAlAs Laser Diode-Array in an External Talbot Cavity," Applied Physics Letters, 58(23), 2586-2588 (1991).

[5] J. R. Leger, and G. Mowry, "External Diode-Laser-Array Cavity with Mode-Selecting Mirror," Applied Physics Letters, 63(21), 2884-2886 (1993).

[6] R. K. Huang, B. Chann, L. J. Missagia, S. J. Augst, R. B. Swint, J. P. Donnelly, A. Sanchez-Rubio, and G. W. Turner, "High-Power Coherent Beam Combination of Semiconductor Laser Arrays." paper CMN1.

[7] I. Hassiaoui, N. Michel, G. Bourdet, R. Mc Bride, M. Lecomte, O. Parillaud, M. Calligaro, M. Krakowski, and J. P. Huignard, "Very compact external cavity diffraction-coupled tapered laser diodes," Applied Optics, 47(6), 746-750 (2008).

[8] B. Liu, Y. Liu, and Y. Braiman, "Coherent addition of high power laser diode array with a V-shape external Talbot cavity,” Opt. Express, 16(25), 20935-20942 (2008).

[9] G. L. Bourdet, I. Hassiaoui, R. McBride, J. F. Monjardin, H. Baker, N. Michel, and M. Krakowski, "Highpower, low-divergence, linear array of quasi-diffraction-limited beams supplied by tapered diodes," Applied Optics, 46(25), 6297-6301 (2007).

[10] J. K. Butler, D. E. Ackley, and D. Botez, "Coupled-Mode Analysis of Phase-Locked Injection-Laser Arrays," Applied Physics Letters, 44(3), 293-295 (1984).

[11] H. F. Talbot, "Facts relating to optical Science," Philosophical Magazine, 9(56), 401-407 (1836).

[12] M. Born, and E. Wolf, [Principles of optics : electromagnetic theory of propagation, interference and diffraction of light] Cambridge University Press, Cambridge ; New York(1999).

[13] J. J. Lim, T. M. Benson, and E. C. Larkins, "Design of wide-emitter single-mode laser diodes," IEEE Journal of Quantum Electronics, 41(4), 506-516 (2005). 

stabilization of extended-cavity tapered lasers with volume Bragg gratings," Applied Physics B-Lasers and Optics, 91(3-4), 493-498 (2008).

[15] D. Paboeuf, G. Lucas-Leclin, P. Georges, N. Michel, M. Krakowski, J. Lim, S. Sujecki, and E. Larkins, "Narrow-line coherently combined tapered laser diodes in a Talbot external cavity with a volume Bragg grating,” Applied Physics Letters, 93(21), 211102 (2008). 